\title{
Black hole collapse simulated by vacuum fluctuations with moving semi-transparent mirror
}

\author{
Jaume Haro ${ }^{1, *}$ and Emilio Elizalde ${ }^{2, \dagger}$ \\ ${ }^{1}$ Departament de Matemàtica Aplicada I, Universitat Politècnica de Catalunya, Diagonal 647, 08028 Barcelona, Spain \\ ${ }^{2}$ Instituto de Ciencias del Espacio (CSIC) \& Institut d'Estudis Espacials de Catalunya (IEEC/CSIC) \\ Campus UAB, Facultat de Ciències, Torre C5-Parell-2a planta, 08193 Bellaterra (Barcelona) Spain
}

(Dated: November 6, 2007)

\begin{abstract}
Creation of scalar massless particles in two-dimensional Minkowski space-time-as predicted by the dynamical Casimir effect-is studied for the case of a semitransparent mirror initially at rest, then accelerating for some finite time, along a trajectory that simulates a black hole collapse (defined by Walker, and Carlitz and Willey), and finally moving with constant velocity. When the reflection and transmission coefficients are those in the model proposed by Barton, Calogeracos, and Nicolaevici $[r(w)=-i \alpha /(\omega+i \alpha)$ and $s(w)=\omega /(\omega+i \alpha)$, with $\alpha \geq 0$ ], the Bogoliubov coefficients on the back side of the mirror can be computed exactly. This allows us to prove that, when $\alpha$ is very large (case of an ideal, perfectly reflecting mirror) a thermal emission of scalar massless particles obeying Bose-Einstein statistics is radiated from the mirror (a black body radiation), in accordance with results previously obtained in the literature. However, when $\alpha$ is finite (semitransparent mirror, a physically realistic situation) the striking result is obtained that the thermal emission of scalar massless particles obeys Fermi-Dirac statistics. We also show here that the reverse change of statistics takes place in a bidimensional fermionic model for massless particles, namely that the Fermi-Dirac statistics for the completely reflecting situation will turn into the Bose-Einstein statistics for a partially reflecting, physical mirror.
\end{abstract}

PACS numbers: 03.70.+k, 04.62.+v

\section{INTRODUCTION}

The Davies-Fulling model [1, 2] describes the creation of scalar massless particles by a moving perfect mirror following a prescribed trajectory. This phenomenon is also termed as the dynamical Casimir effect. Recently, the authors of the present paper introduced a Hamiltonian formulation in order to address some problems associated with the physical description of this effect in the time interval while the mirror is moving [3, 4]; in particular, of the regularization procedure, which turns out to be decisive for the correct derivation of physically meaningful quantities. A basic difference with previous results was that the motion force derived within the new approach contains a reactive term-proportional to the mirror's acceleration. This term is of the essence in order to obtain particles with a positive energy all the time while the oscillation of the mirror takes place, and which always satisfy the energy conservation law. Those result followed essentially from the introduction of physically realistic conditions, e.g. a semi-transparent or partially transmitting mirror, which is perfectly reflecting for low frequencies but becomes transparent to very high ones.

Here we will study a different aspect of the introduction of physically plausible, semitransparent mirrors, namely the particle spectrum produced —in the conditions of the Fulling-Davies effect—by a mirror of this sort which is initially at rest, then accelerates during a large enough (but finite) time span, $u_{0}$, along a trajectory that simulates a black hole collapse, as defined by Walker [5], and Carlitz and Willey [6]:

$$
v=\frac{1}{k}\left(1-e^{-k u}\right)
$$

(in light-like coordinates, where $k$ is some frequency), and finally, for $u \geq u_{0}$, is left alone moving with constant velocity in an inertial trajectory.

\footnotetext{
*E-mail: jaime.haro@upc.edu

† E-mail: elizalde@ieec.uab.es, elizalde@math.mit.edu
} 
We will be interested in calculating the radiation emitted by the mirror from its back (e.g. right) side. As is well-known, a perfect mirror that follows this kind of trajectory produces a thermal emission of scalar massless particles obeying BoseEinstein statistics. More precisely, for $1 \ll \omega^{\prime} / k \ll e^{k u_{0}}$ and $1 \ll \omega^{\prime} / \omega \ll e^{k u_{0}}$, one has [7, 8, 9]

$$
\left|\beta_{\omega, \omega^{\prime}}^{R, R}\right|^{2} \equiv\left|\left(\phi_{\omega, R}^{\text {out }} * \phi_{\omega^{\prime}, R}^{\text {in }}\right)\right|^{2} \cong \frac{1}{2 \pi \omega^{\prime} k}\left(e^{2 \pi \omega / k}-1\right)^{-1} .
$$

Turning to the case of a partially reflecting mirror — in which we will be mainly interested in this paper —in order to obtain the radiation on its right hand side (rhs), we also need to calculate the Bogoliubov coefficient: $\beta_{\omega, \omega^{\prime}}^{R, L} \equiv\left(\phi_{\omega, R}^{\text {out }}{ }^{*} ; \phi_{\omega^{\prime}, L}^{\text {in }}\right)^{*}$.

We thus first obtain the 'in' modes on the rhs of the mirror when the reflection and transmission coefficients are $r(w)=\frac{-i \alpha}{\omega+i \alpha}$ and $s(w)=\frac{\omega}{\omega+i \alpha}$, with $\alpha \geq 0$, that is, when the Lagrangian density is given by [10, 11, 12]

$$
\mathcal{L}=\frac{1}{2}\left[\left(\partial_{t} \phi\right)^{2}-\left(\partial_{z} \phi\right)^{2}\right]-\alpha \sqrt{1-\dot{g}^{2}(t)} \phi^{2} \delta(z-g(t)),
$$

where $z=g(t)$ is the trajectory in the $(t, z)$ coordinates.

\section{A. The main results}

We now state the main results that will be obtained in this paper (for $1 \ll \omega^{\prime} / k \ll e^{k u_{0}}$ and $1 \ll \omega^{\prime} / \omega \ll e^{k u_{0}}$ ).

1. For a perfectly reflecting mirror, i.e., when $\omega^{\prime} \ll \alpha$, we will see that

$$
\left|\beta_{\omega, \omega^{\prime}}^{R, R}\right|^{2} \cong \frac{1}{2 \pi \omega^{\prime} k}\left(e^{2 \pi \omega / k}-1\right)^{-1}, \quad\left|\beta_{\omega, \omega^{\prime}}^{R, L}\right|^{2} \cong 0,
$$

namely, a thermal radiation of massless particles obeying Bose-Einstein statistics is produced.

2. For a perfectly transparent mirror, i.e.,when $\alpha \cong 0$, we will get that

$$
\left|\beta_{\omega, \omega^{\prime}}^{R, R}\right|^{2} \cong 0, \quad\left|\beta_{\omega, \omega^{\prime}}^{R, L}\right|^{2} \cong 0 .
$$

In other words, there is no particle production.

3. In the physically more realistic case of a partially transmitting mirror (transparent to high enough frequencies [3]), i.e., when $\alpha \ll \omega^{\prime}$, what we obtain is

$$
\begin{aligned}
& \left|\beta_{\omega, \omega^{\prime}}^{R, R}\right|^{2} \cong \frac{1}{2 \pi \omega k}\left(\frac{\alpha}{\omega^{\prime}}\right)^{2}\left(e^{2 \pi \omega / k}+1\right)^{-1}, \\
& \left|\beta_{\omega, \omega^{\prime}}^{R, L}\right|^{2} \sim \frac{1}{\omega \omega^{\prime}} \mathcal{O}\left[\left(\frac{\alpha}{\omega^{\prime}}\right)^{2}\right] .
\end{aligned}
$$

And, since $\left|\beta_{\omega, \omega^{\prime}}^{R, L}\right| \ll\left|\beta_{\omega, \omega^{\prime}}^{R, R}\right|$, we then conclude that a semitransparent mirror emits a thermal radiation of scalar massless particles obeying Fermi-Dirac statistics.

Moreover, Eq. (6) does show that there are no ultraviolet divergences when the mirror is semi-transparent (in agreement with previous conclusions in [3, 4, 13]) and, consequently, the number of produced particles in the $\omega$ mode, namely $\mathcal{N}_{\omega}$, is finite. In fact, with good approximation we have calculated that

$$
\mathcal{N}_{\omega} \cong \frac{1}{2 \pi \omega}\left(\frac{\alpha}{k}\right)^{2}\left(e^{2 \pi \omega / k}+1\right)^{-1}
$$

It must be here remarked that this phenomenon will in no way occur for a perfectly reflecting mirror, where the number of produced particles in a prescribed mode diverges linearly with time [8, 9].

Actually, the same kind of effect—but now reversed—occurs if a bidimensional fermionic model of massless particles is considered, where in the perfectly reflecting case the mirror emits a thermal radiation of fermions obeying the FermiDirac statistics. When the mirror becomes semi-transparent (the physically realistic case), the emitted thermal radiation will obey Bose-Einstein statistics. We interpret these results as a proof of the fact that the spectrum of the radiation 
produced by a mirror which follows a trajectory that simulates black hole collapse does not depend on the statistics of the field, being just determined by the interaction of the mirror with the radiation field. In our case this interaction is given by the reflection and transmission coefficients which depend on the parameter $\alpha$, that determines the spectrum of the emitted radiation.

Here it is important to emphasize that the word 'statistics' refers all the time to the $\beta$-Bogoliubov coefficient characterizing the spectrum of the radiated particles and not to the algebra obeyed by the creation and annihilation operators, that always satisfy the corresponding canonical commutation relations (anti-commutation, in the fermionic case). That is, e.g. in the second case studied the original particles continue to be fermions, but the spectrum of the radiated emission corresponds to bosonic ones, when the mirror becomes physical, that is, semi-transparent. As a consequence, fermionic number is not actually violated.

Related phenomenons of similar kind have been reported to occur also in other situations:

1. In the case of an electric charge following the trajectory $v=\frac{1}{k}\left(1-e^{-k u}\right)$. When the radiation field has spin 1 , the radiation emitted by the charge obeys Bose-Einstein statistics, but when a scalar charge, and consequently an scalar radiation field, is considered, the emitted radiation will obey Fermi-Dirac statistics (see [14] for more details).

2. When measuring the spectrum of a scalar field by using a DeWitt detector which follows a uniformly accelerated world-line in Minkowski space-time, one can show that, when the dimension of the space-time is even the BoseEinstein statistics is obtained; however when this dimension is odd the reverse change of statistics occurs (see [15] for further details).

Finally, in an Appendix we will specify some sufficient conditions in order to ensure the convergence of the total number of produced particles and of their associated energy.

\section{PERFECTLY REFLECTING, MOVING MIRROR}

Consider a massless scalar field $\phi$ in two-dimensional Minkowski space-time interacting with a moving mirror. Assume that the mirror trajectory simulates a black hole collapse [2, 16], this is, that it reduces to the following form in the lightlike coordinates $u \equiv t-z$ and $v \equiv t+z$ :

$$
v=V(u) \equiv\left\{\begin{array}{ccc}
u & \text { if } \quad u \leq 0 \\
\frac{1}{k}\left(1-e^{-k u}\right) & \text { if } & 0 \leq u \leq u_{0} \\
V\left(u_{0}\right)+A\left(u-u_{0}\right) & \text { if } & u \geq u_{0},
\end{array}\right.
$$

with $A=e^{-k u_{0}}$, where $k$ is a frequency and $u_{0} \gg 1$. Note that this trajectory can also be written as follows

$$
u=U(v) \equiv\left\{\begin{array}{ccc}
v & \text { if } \quad v \leq 0 \\
-\frac{1}{k} \ln (1-k v) & \text { if } 0 \leq v \leq v_{0} \\
U\left(v_{0}\right)+A^{-1}\left(v-v_{0}\right) & \text { if } & v \geq v_{0} .
\end{array}\right.
$$

For a perfectly reflecting mirror, the sets of 'in' and 'out' mode functions are [17]

$$
\left\{\begin{array}{l}
\phi_{\omega, R}^{i n}(u, v)=\frac{1}{\sqrt{4 \pi|\omega|}}\left(e^{-i \omega v}-e^{-i \omega V(u)}\right) \theta(v-V(u)) \\
\phi_{\omega, L}^{i n}(u, v)=\frac{1}{\sqrt{4 \pi|\omega|}}\left(e^{-i \omega u}-e^{-i \omega U(v)}\right) \theta(u-U(v))
\end{array}\right.
$$

and

$$
\left\{\begin{array}{l}
\phi_{\omega, R}^{o u t}(u, v)=\frac{1}{\sqrt{4 \pi|\omega|}}\left(e^{-i \omega u}-e^{-i \omega U(v)}\right) \theta(v-V(u)) \\
\phi_{\omega, L}^{o u t}(u, v)=\frac{1}{\sqrt{4 \pi|\omega|}}\left(e^{-i \omega v}-e^{-i \omega V(u)}\right) \theta(u-U(v)),
\end{array}\right.
$$


respectively. Our main objective in this section will be to calculate the beta Bogoliubov coefficient

$$
\beta_{\omega, \omega^{\prime}}^{R, R} \equiv\left(\phi_{\omega, R}^{\text {out }}{ }^{*} ; \phi_{\omega^{\prime}, R}^{\text {in }}\right)^{*}, \quad \text { with } \quad \omega, \omega^{\prime}>0,
$$

where the brackets on the rhs denote the usual product for scalar fields (see, e.g., Birrell-Davies [16]). In order to compute this coefficient we choose the right null future infinity domain, $\mathcal{J}_{R}^{+}$. There, we have

$$
\begin{aligned}
\beta_{\omega, \omega^{\prime}}^{R, R}=2 i \int_{\mathbb{R}} d u \phi_{\omega, R}^{\text {out }} \partial_{u} \phi_{\omega^{\prime}, R}^{i n}= & \frac{1}{2 \pi i \sqrt{\omega \omega^{\prime}}} \frac{\omega^{\prime}}{\omega+\omega^{\prime}}-\frac{1}{2 \pi i \sqrt{\omega \omega^{\prime}}} e^{-i \omega u_{0}} e^{-i \omega^{\prime} V\left(u_{0}\right)} \frac{\omega^{\prime} A}{\omega+\omega^{\prime} A} \\
& -\frac{1}{2 \pi k} \sqrt{\frac{\omega^{\prime}}{\omega}} \int_{0}^{1-A} d s(1-s)^{i \omega / k} e^{-i \omega^{\prime} / k s} .
\end{aligned}
$$

Taking into account that $1 \ll \omega^{\prime} / k \ll A^{-1}$ and $1 \ll \omega^{\prime} / k \ll A^{-1}$, we get with good approximation

$$
\beta_{\omega, \omega^{\prime}}^{R, R} \cong \frac{1}{2 \pi i \sqrt{\omega \omega^{\prime}}}-\frac{1}{2 \pi k} \sqrt{\frac{\omega^{\prime}}{\omega}} \int_{0}^{1-A} d s(1-s)^{i \omega / k} e^{-i \omega^{\prime} / k s} .
$$

To obtain an explicit expression for the second term on the rhs, we consider the domain

$$
D \equiv\left\{z \in \mathbb{C} \mid \operatorname{Re} z \in[0,1-A], \operatorname{Im} z \in[-\epsilon, 0] \text {, with } k / w^{\prime} \ll \epsilon \ll 1\right\}
$$

and going through the same steps as in [9], we easily obtain

$$
\beta_{\omega, \omega^{\prime}}^{R, R} \cong \frac{1}{2 \pi i \sqrt{\omega \omega^{\prime}}} e^{-i \omega^{\prime} / k}\left(\frac{i k}{\omega^{\prime}}\right)^{i \omega / k} \Gamma(1+i \omega / k) .
$$

As a consequence, using that $|\Gamma(1+i \omega / k)|^{2}=\frac{\pi \omega / k}{\sinh (\pi \omega / k)}$ (see [18]) we get the announced result, that for a perfect reflecting mirror the relevant Bogoliubov coefficient is

$$
\left|\beta_{\omega, \omega^{\prime}}^{R, R}\right|^{2} \cong \frac{1}{2 \pi \omega^{\prime} k}\left(e^{2 \pi \omega / k}-1\right)^{-1}
$$

\section{PARTIALLY REFLECTING, MOVING MIRROR}

Now we start by reducing the problem to co-moving coordinates $(\tau, \rho)$, that is, those for which the mirror remains at rest, $\tau$ being the proper time of the mirror, and we take $\rho$ such that its trajectory be given by $\rho=0$. Introducing the light-like coordinates $(\bar{u}, \bar{v})$, defined as

$$
\bar{u} \equiv \tau-\rho ; \quad \bar{v} \equiv \tau+\rho,
$$

we will calculate the mirror's trajectory in the coordinates $(\bar{u}, \bar{v})$. Along this trajectory, the length element obeys the identity [19]

$$
d \tau^{2}=d \bar{u}^{2}=d \bar{v}^{2}=V^{\prime}(u) d u^{2}=U^{\prime}(v) d v^{2} .
$$

An easy calculation yields then the relations

$$
\bar{v}=\bar{u}(u) \equiv\left\{\begin{array}{cc}
u & \text { if } \quad u \leq 0 \\
\frac{2}{k}\left(1-e^{-k u / 2}\right) & \text { if } 0 \leq u \leq u_{0} \\
\bar{u}\left(u_{0}\right)+\sqrt{A}\left(u-u_{0}\right) & \text { if } \quad u \geq u_{0},
\end{array}\right.
$$

and

$$
\bar{u}=\bar{v}(v) \equiv\left\{\begin{array}{cc}
v & \text { if } \quad v \leq 0 \\
\frac{2}{k}(1-\sqrt{1-k v}) & \text { if } 0 \leq v \leq v_{0} \\
\bar{v}\left(v_{0}\right)+A^{-1 / 2}\left(v-v_{0}\right) & \text { if } \quad v \geq v_{0} .
\end{array}\right.
$$


When the semitransparent mirror is at rest, scattering is described by the analytical $S$-matrix (see [4, 20] for full details)

$$
S(\omega)=\left(\begin{array}{cc}
s(\omega) & r(\omega) e^{-2 i \omega L} \\
r(\omega) e^{2 i \omega L} & s(\omega)
\end{array}\right)
$$

where $x=L$ is the position of the mirror. The $S$ matrix is taken to be real in the temporal domain, causal, unitary, and the identity at high frequencies [20], being $r(\omega)$ and $s(\omega)$ the reflection and transmission functions, which are analytic and such that tend in modulus to -1 and 0 , respectively, as $\omega \rightarrow 0$ (to 0 and 1 , as $\omega \rightarrow \infty$ ). The 'in' modes in the coordinates $(\bar{u}, \bar{v})$ are [10]

$$
\begin{aligned}
& g_{\omega, R}^{i n}(\bar{u}, \bar{v})=\frac{1}{\sqrt{4 \pi|\omega|}} s(\omega) e^{-i \omega \bar{v}} \theta(\bar{u}-\bar{v})+\frac{1}{\sqrt{4 \pi|\omega|}}\left[e^{-i \omega \bar{v}}+r(\omega) e^{-i \omega \bar{u}}\right] \theta(\bar{v}-\bar{u}), \\
& g_{\omega, L}^{i n}(\bar{u}, \bar{v})=\frac{1}{\sqrt{4 \pi|\omega|}}\left[e^{-i \omega \bar{u}}+r(\omega) e^{-i \omega \bar{v}}\right] \theta(\bar{u}-\bar{v})+\frac{1}{\sqrt{4 \pi|\omega|}} s(\omega) e^{-i \omega \bar{u}} \theta(\bar{v}-\bar{u}) .
\end{aligned}
$$

Note that the 'in' modes in the coordinates $(u, v)$, namely $\phi^{i n}$, are defined in the right null past infinity $\mathcal{J}_{R}^{-}$by

$$
\phi_{\omega, R}^{i n}=\frac{1}{\sqrt{4 \pi|\omega|}} e^{-i \omega v}, \quad \phi_{\omega, L}^{i n}=0,
$$

and, in the left null past infinity $\mathcal{J}_{L}^{-}$, by

$$
\phi_{\omega, R}^{i n}=0, \quad \phi_{\omega, L}^{i n}=\frac{1}{\sqrt{4 \pi|\omega|}} e^{-i \omega u} .
$$

From these definitions it is clear that $\bar{g}_{\omega, k}^{i n}(u, v) \equiv g_{\omega, k}^{i n}(\bar{u}(u), \bar{v}(v))$ with $k=R, L$ are not such modes. However, the modes $\bar{g}_{\omega, k}^{i n}$ do actually constitute an orthonormal basis of the space of solutions of our problem. As a consequence, if we use the fact that $\bar{g}_{-\omega, k}^{i n}=\bar{g}_{\omega, k}^{i n *}$, we can obtain the following relation

$$
\phi_{\omega, k}^{i n}=\int_{\mathbb{R}} d \omega^{\prime} \chi\left(\omega^{\prime}\right)\left(\bar{g}_{\omega^{\prime}, k}^{i n} ; \phi_{\omega, k}^{i n}\right) \bar{g}_{\omega^{\prime}, k}^{i n}, \quad k=R, L
$$

with $\chi\left(\omega^{\prime}\right)$ the sign function. To be remarked is that Eq. 27) is to be interpreted as follows

$$
\phi_{\omega, k}^{i n}=\lim _{\lambda \rightarrow \infty} \int_{\mathbb{R}} d \omega^{\prime} \chi\left(\omega^{\prime}\right)\left(\bar{g}_{\omega^{\prime}, k}^{i n} ; \phi_{\omega, k}^{i n}\right) \bar{g}_{\omega^{\prime}, k}^{i n} F_{\lambda}\left(\omega^{\prime}\right)
$$

being $F_{\lambda}\left(\omega^{\prime}\right)$ a frequency cut-off, as for instance $\frac{\lambda^{2}}{\lambda^{2}+\left(\omega^{\prime}\right)^{2}}$.

To calculate the 'in' modes explicitly, we have chosen the coefficients $r(w)=\frac{-i \alpha}{\omega+i \alpha}$ and $s(w)=\frac{\omega}{\omega+i \alpha}$ with $\alpha \geq 0$. In this case, on the rhs of the mirror we obtain

$$
\phi_{\omega, R}^{i n}(u, v)=\frac{1}{\sqrt{4 \pi|\omega|}} e^{-i \omega v}+\phi_{\omega, R}^{r e f l}(u) ; \quad \phi_{\omega, L}^{i n}(u, v)=\phi_{\omega, L}^{\text {trans }}(u),
$$

where

$$
\phi_{\omega, R}^{r e f l}(u)=\left\{\begin{array}{cc}
\frac{1}{\sqrt{4 \pi|\omega|}} \frac{-i \alpha}{\omega+i \alpha} e^{-i \omega V(u)} ; & u \leq 0 \\
\frac{1}{\sqrt{4 \pi|\omega|}} \frac{-i \alpha}{\omega+i \alpha} e^{-\alpha \bar{u}(u)}-\frac{2 \alpha}{k \sqrt{4 \pi|\omega|}} e^{-i \frac{\omega}{k}} \int_{0}^{\frac{k}{2} \bar{u}(u)} d s e^{\frac{i \omega}{k}\left(s+1-\frac{k}{2} \bar{u}(u)\right)^{2}} e^{-\frac{2 \alpha s}{k}} ; & 0 \leq u \leq u_{0} \\
\frac{1}{\sqrt{4 \pi|\omega|}} \frac{-i \alpha}{\omega+i \alpha} e^{-\alpha \bar{u}(u)}-\frac{1}{\sqrt{4 \pi|\omega|}} \frac{i \alpha}{\sqrt{A \omega+i \alpha}}\left[e^{-i \omega V(u)}-e^{-i \omega V\left(u_{0}\right)} e^{-\alpha\left(\bar{u}(u)-\bar{u}\left(u_{0}\right)\right)}\right] & \\
-\frac{2 \alpha}{k \sqrt{4 \pi|\omega|}} e^{-i \frac{\omega}{k}} e^{-\alpha\left(\bar{u}(u)-\bar{u}\left(u_{0}\right)\right)} \int_{0}^{\frac{k}{2} \bar{u}\left(u_{0}\right)} d s e^{\frac{i \omega}{4}\left(s+1-\frac{k}{2} \bar{u}\left(u_{0}\right)\right)^{2}} e^{-\frac{2 \alpha s}{k}} ; & u \geq u_{0}
\end{array}\right.
$$


and

$$
\phi_{\omega, L}^{\text {trans }}(u)=\left\{\begin{array}{cc}
\frac{1}{\sqrt{4 \pi|\omega|}} \frac{\omega}{\omega+i \alpha} e^{-i \omega V(u)} ; & u \leq 0 \\
\frac{1}{\sqrt{4 \pi|\omega|}} e^{-i \omega u}+\frac{1}{\sqrt{4 \pi|\omega|}} \frac{-i \alpha}{\omega+i \alpha} e^{-\alpha \bar{u}(u)}-\frac{2 \alpha}{k \sqrt{4 \pi|\omega|}} \int_{0}^{\frac{k}{2} \bar{u}(u)} d s\left(s+1-\frac{k}{2} \bar{u}(u)\right)^{2 i \frac{\omega}{k}} e^{-\frac{2 \alpha s}{k}} ; & 0 \leq u \leq u_{0} \\
\frac{1}{\sqrt{4 \pi|\omega|}} \frac{-i \alpha}{\omega+i \alpha} e^{-\alpha \bar{u}(u)}+\frac{1}{\sqrt{4 \pi|\omega|}} \frac{e^{-i \omega u_{0}}}{\omega+i \alpha \sqrt{A}}\left[\omega e^{-i \frac{\omega}{\sqrt{A}}\left(\bar{u}(u)-\bar{u}\left(u_{0}\right)\right)}+i \alpha \sqrt{A} e^{-\alpha\left(\bar{u}(u)-\bar{u}\left(u_{0}\right)\right)}\right] & u \geq u_{0} \\
-\frac{2 \alpha}{k \sqrt{4 \pi|\omega|}} e^{-\alpha\left(\bar{u}(u)-\bar{u}\left(u_{0}\right)\right)} \int_{0}^{\frac{k}{2} \bar{u}\left(u_{0}\right)} d s\left(s+1-\frac{k}{2} \bar{u}\left(u_{0}\right)\right)^{2 i \frac{\omega}{k}} e^{-\frac{2 \alpha s}{k}} ; & u
\end{array}\right.
$$

Note that (as already advanced) in the case of perfect reflection, that is when $\alpha \rightarrow \infty$, we get

$$
\phi_{\omega, R}^{r e f l}(u) \rightarrow-\frac{1}{\sqrt{4 \pi|\omega|}} e^{-i \omega V(u)}, \quad \phi_{\omega, L}^{\text {trans }}(u) \rightarrow 0,
$$

and when the mirror is transparent, i.e., when $\alpha \rightarrow 0$, we have

$$
\phi_{\omega, R}^{r e f l}(u) \rightarrow 0, \quad \phi_{\omega, L}^{\text {trans }}(u) \rightarrow \frac{1}{\sqrt{4 \pi|\omega|}} e^{-i \omega u} .
$$

We are interested in the particle production on the rhs of the mirror, for this reason we must now obtain, for $\omega, \omega^{\prime}>0$

$$
\beta_{\omega, \omega^{\prime}}^{R, R} \equiv\left(\phi_{\omega, R}^{o u t}{ }^{*} ; \phi_{\omega^{\prime}, R}^{\text {in }}\right)^{*}, \quad \text { and } \quad \beta_{\omega, \omega^{\prime}}^{R, L} \equiv\left(\phi_{\omega, R}^{\text {out }} * \phi_{\omega^{\prime}, L}^{\text {in }}\right)^{*} .
$$

In order to calculate these products we better choose the right null infinity $\mathcal{J}_{R}^{+}$, because here the 'out' modes acquire a simple form, namely

$$
\beta_{\omega, \omega^{\prime}}^{R, R}=\left(\phi_{\omega, R}^{\text {out }}{ }^{*} ; \phi_{\omega^{\prime}, R}^{\text {refl }}\right)^{*}, \quad \text { and } \quad \beta_{\omega, \omega^{\prime}}^{R, L} \equiv\left(\phi_{\omega, R}^{\text {out }}{ }^{*} ; \phi_{\omega^{\prime}, L}^{\text {trans }}\right)^{*} .
$$

We start by calculating $\beta_{\omega, \omega^{\prime}}^{R, R}=2 i \int_{\mathbb{R}} d u \phi_{\omega, R}^{o u t} \partial_{u} \phi_{\omega^{\prime}, R}^{r e f l}$, with the result

$$
\begin{aligned}
& \beta_{\omega, \omega^{\prime}}^{R, R} \cong \frac{1}{2 \pi \sqrt{\omega \omega^{\prime}}} \frac{\alpha}{\omega^{\prime}+i \alpha}\left[1-\frac{\alpha}{k} \int_{A}^{1} d x x^{i \omega / k-1 / 2} e^{-2 \alpha(1-\sqrt{x}) / k}\right] \\
& +\frac{\alpha}{2 \pi k i \sqrt{\omega \omega^{\prime}}} e^{-i \omega^{\prime} / k} \int_{A}^{1} d x x^{i \omega / k-1 / 2} e^{i \omega^{\prime} x / k}\left[1-\frac{2 \alpha}{k} \int_{0}^{1-\sqrt{x}} e^{i \omega^{\prime}\left(s^{2}+2 s \sqrt{x}\right) / k} e^{-2 \alpha s / k}\right] .
\end{aligned}
$$

Now, provided that $\omega^{\prime} \ll \alpha$, Eq. (34) turns into Eq. (14). As a consequence, we precisely obtain the same behavior as for a perfectly reflecting mirror. However, in the case $\alpha \ll \omega^{\prime}$,

$$
\beta_{\omega, \omega^{\prime}}^{R, R} \cong \frac{\alpha}{2 \pi k i \sqrt{\omega \omega^{\prime}}} e^{-i \omega^{\prime} / k}\left(i \frac{k}{\omega^{\prime}}\right)^{i \omega / k+1 / 2} \Gamma(1 / 2+i \omega / k),
$$

and using the identity $|\Gamma(1 / 2+i \omega / k)|^{2}=\frac{\pi}{\cosh (\pi \omega / k)}$ (see [18]), we conclude that

$$
\left|\beta_{\omega, \omega^{\prime}}^{R, R}\right|^{2} \cong \frac{1}{2 \pi k \omega}\left(\frac{\alpha}{\omega^{\prime}}\right)^{2}\left(e^{2 \pi \omega / k}+1\right)^{-1} .
$$

Finally, a simple but rather cumbersome calculation yields the results

$$
\left|\beta_{\omega, \omega^{\prime}}^{R, L}\right|^{2} \cong 0, \quad \omega^{\prime} \ll \alpha,
$$

and

$$
\left|\beta_{\omega, \omega^{\prime}}^{R, L}\right|^{2} \sim \frac{1}{\omega \omega^{\prime}} \mathcal{O}\left[\left(\frac{\alpha}{\omega^{\prime}}\right)^{2}\right], \quad \alpha \ll \omega^{\prime}
$$


Note that in the case $\alpha \ll \omega^{\prime}$ we indeed obtain the nice feature that the number of created particles in the $\omega$ mode, together with the radiated energies, are both finite quantities when $u_{0} \rightarrow \infty$, in perfect agreement with the conclusions in [8]. More precisely, for a partially transmitting mirror the number of produced particles in the $\omega$ mode

$$
\mathcal{N}_{\omega} \equiv \int_{0}^{\infty} d \omega^{\prime}\left(\left|\beta_{\omega, \omega^{\prime}}^{R, R}\right|^{2}+\left|\beta_{\omega, \omega^{\prime}}^{R, L}\right|^{2}\right)
$$

is approximately $\int_{0}^{\infty} d \omega^{\prime}\left|\beta_{\omega, \omega^{\prime}}^{R, R}\right|^{2}$. In order to calculate this quantity, we split the domain $[0, \infty)$ into two disjoints sets, $[0, k)$ and $[k, \infty)$, respectively. In the second domain we can carry out the approximation (36) to obtain

$$
\int_{k}^{\infty} d \omega^{\prime}\left|\beta_{\omega, \omega^{\prime}}^{R, R}\right|^{2} \cong \frac{1}{2 \pi \omega}\left(\frac{\alpha}{k}\right)^{2}\left(e^{2 \pi \omega / k}+1\right)^{-1} .
$$

In the other domain, assuming that $k \ll 1$, we have $\omega^{\prime} \ll 1$ and thus for incident waves of very low frequency the mirror behaves like a perfect reflector. For this reason we can use the formula (14) and a simple calculation yields

$$
\int_{0}^{k} d \omega^{\prime}\left|\beta_{\omega, \omega^{\prime}}^{R, R}\right|^{2} \sim \mathcal{O}\left(\frac{k^{2}}{\omega\left(\omega^{2}+k^{2}\right)}\right) .
$$

Thus, since $k \ll 1$, we conclude that the number of produced particles in the $\omega$ mode is approximately

$$
\mathcal{N}_{\omega} \cong \frac{1}{2 \pi \omega}\left(\frac{\alpha}{k}\right)^{2}\left(e^{2 \pi \omega / k}+1\right)^{-1}
$$

and the radiated energy $\mathcal{E} \equiv \int_{0}^{\infty} d \omega \hbar \omega \mathcal{N}_{\omega}$ is, also with good approximation,

$$
\mathcal{E} \cong \frac{\hbar \alpha^{2}}{4 \pi^{2} k} \ln 2
$$

This completes the proof of all of the statements above.

It is appropriate to remark that there is a crucial difference with the case $\omega^{\prime} \ll \alpha$, where the number of radiated particles in the $\omega$ mode diverges logarithmically with $u_{0} \rightarrow \infty$. In this situation the physically relevant quantity is the number of created particles in the $\omega$ mode per unit time $t_{0} \equiv \frac{1}{2}\left(u_{0}+V\left(u_{0}\right)\right) \cong \frac{1}{2} u_{0}$. This dimensionless quantity is finite and its value is given by [8, 9]

$$
\lim _{t_{0} \rightarrow \infty} \frac{1}{t_{0}} \mathcal{N}_{\omega}=\frac{1}{\pi}\left(e^{2 \pi \omega / k}-1\right)^{-1} .
$$

Finally, it is also interesting to calculate the detector response function [16], namely $\mathcal{F}(\omega)$, for an inertial DeWitt detector following the trajectory $z=0$. This function is given by

$$
\mathcal{F}(\omega) \equiv \int_{\mathbb{R}} d t \int_{\mathbb{R}} d t^{\prime} e^{-i \omega\left(t-t^{\prime}\right)}\left\langle 0, i n\left|\phi(0, t) \phi\left(0, t^{\prime}\right)\right| 0, i n\right\rangle,
$$

where $\mid 0$, in $\rangle$ denotes the 'in' vacuum state. This function is related to the average number of produced particles in the $\omega$-mode, through the relation [5]

$$
\mathcal{F}(\omega)=\frac{\pi}{\omega} \mathcal{N}_{\omega}
$$

Then, for a partially transmitting mirror, from Eq. 41, we conclude that

$$
\mathcal{F}(\omega) \cong \frac{1}{2 \omega^{2}}\left(\frac{\alpha}{k}\right)^{2}\left(e^{2 \pi \omega / k}+1\right)^{-1}
$$

However, for a perfect reflecting mirror, this quantity diverges. In this case the relevant function is the detector response function per unit time [15], namely $\mathcal{P}(\omega) \equiv \lim _{t_{0} \rightarrow \infty} \frac{1}{t_{0}} \mathcal{F}(\omega)$. Using (43), one then obtains the Planckian spectrum

$$
\mathcal{P}(\omega)=\frac{1}{\omega}\left(e^{2 \pi \omega / k}-1\right)^{-1} .
$$




\section{CASE OF THE DIRAC FIELD}

In this section we consider the Dirac equation, in $1+1$ dimensions, for a massless field

$$
\gamma^{0} \partial_{t} \psi+\gamma^{1} \partial_{x} \psi=0
$$

where here the Dirac matrices are (see [21])

$$
\gamma^{0}=\left(\begin{array}{ll}
0 & 1 \\
1 & 0
\end{array}\right) \quad \gamma^{1}=\left(\begin{array}{cc}
0 & -1 \\
1 & 0
\end{array}\right)
$$

In the variables $(u, v)$, Eq. (48) is

$$
\left(\begin{array}{ll}
0 & 1 \\
0 & 0
\end{array}\right) \partial_{u} \psi+\left(\begin{array}{cc}
0 & 0 \\
1 & 0
\end{array}\right) \partial_{v} \psi=0
$$

and we conclude that its general solution can be written as

$$
\psi(u, v)=\left(\begin{array}{l}
F(u) \\
G(v)
\end{array}\right)
$$

Consider now again the trajectory defined in Sect. II. The vector normal to the trajectory is $n_{\mu}=\frac{1}{2 \sqrt{V^{\prime}(u)}}\left(V^{\prime}(u),-1\right)$ and the current vector is given by $j^{\mu}=2\left(|G|^{2},|F|^{2}\right)$. Then, for a perfectly reflecting mirror we must impose that the normal component of the current vanishes on the mirror ([22],[23]), that is, $j^{\mu} n_{\mu}=0$, and thus the condition follows that

$$
V^{\prime}(u)|G|^{2}-|F|^{2}=0
$$

From here we can calculate the corresponding family of 'in and 'out' modes

$$
\begin{aligned}
& \psi_{\omega, R}^{i n}(u, v) \equiv\left[\frac{1}{\sqrt{2 \pi}}\left(\begin{array}{l}
0 \\
1
\end{array}\right) e^{-i \omega v}-\sqrt{\frac{V^{\prime}(u)}{2 \pi}}\left(\begin{array}{l}
1 \\
0
\end{array}\right) e^{-i \omega V(u)}\right] \theta(v-V(u)), \\
& \psi_{\omega, R}^{\text {out }}(u, v) \equiv\left[\frac{1}{\sqrt{2 \pi}}\left(\begin{array}{l}
1 \\
0
\end{array}\right) e^{-i \omega u}-\sqrt{\frac{U^{\prime}(v)}{2 \pi}}\left(\begin{array}{l}
1 \\
0
\end{array}\right) e^{-i \omega U(v)}\right] \theta(v-V(u)) .
\end{aligned}
$$

In this case the beta Bogoliubov coefficient is given by

$$
\beta_{\omega, \omega^{\prime}}^{R, R} \equiv\left(\psi_{\omega, R}^{\text {out } *} ; \psi_{\omega^{\prime}, R}^{\text {in }}\right)^{*}=\int\left(\psi_{\omega, R}^{\text {out }}\right)^{t} \psi_{\omega^{\prime}, R}^{\text {in }},
$$

where $\left(\psi_{\omega, R}^{\text {out }}\right)^{t}$ denotes the transposed of the vector $\psi_{\omega, R}^{\text {out }}$.

Performing the calculation in the right null future infinity domain, $\mathcal{J}_{R}^{+}$, we obtain

$$
\beta_{\omega, \omega^{\prime}}^{R, R} \cong \frac{1}{2 \pi i \omega^{\prime}}-\frac{1}{2 \pi k} \int_{0}^{1-A} d s(1-s)^{i \omega / k-1 / 2} e^{-i \omega^{\prime} s / k}
$$

Then, as in [9], we easily get

$$
\beta_{\omega, \omega^{\prime}}^{R, R} \cong \frac{1}{2 \pi k} e^{-i \omega^{\prime} / k}\left(\frac{i k}{\omega^{\prime}}\right)^{i \omega / k+1 / 2} \Gamma(1 / 2+i \omega / k),
$$

and thus, since $|\Gamma(1 / 2+i \omega / k)|^{2}=\frac{\pi}{\cosh (\pi \omega / k)}$ (see [18]), we finally arrive to the anticipated result that

$$
\left|\beta_{\omega, \omega^{\prime}}^{R, R}\right|^{2} \simeq \frac{1}{2 \pi \omega^{\prime} k}\left(e^{2 \pi \omega / k}+1\right)^{-1} .
$$




\section{A. Partially reflecting, moving mirror}

We start with the two orthonormal basis

$$
\psi_{1, \omega}(v) \equiv \frac{1}{\sqrt{2 \pi}}\left(\begin{array}{l}
0 \\
1
\end{array}\right) \sqrt{\bar{v}^{\prime}(v)} e^{-i \omega \bar{v}(v)}, \quad \psi_{2, \omega}(u) \equiv \frac{1}{\sqrt{2 \pi}}\left(\begin{array}{l}
1 \\
0
\end{array}\right) \sqrt{\bar{u}^{\prime}(u)} e^{-i \omega \bar{u}(u)},
$$

where the functions $\bar{v}$ and $\bar{u}$ are defined in Sect. III. The normal component of the mirror's current given by each of these two functions are $\frac{1}{2 \pi}$ and $-\frac{1}{2 \pi}$, respectively.

It is interesting to observe the analogy between the fermionic and the scalar cases. Note that the current for a scalar field is

$$
j^{\mu}=i\left(\phi \partial_{t} \phi^{*}-\phi^{*} \partial_{t} \phi,-\phi \partial_{x} \phi^{*}+\phi^{*} \partial_{x} \phi\right),
$$

and if we choose the orthonormal basis:

$$
\phi_{1, \omega}(v) \equiv \frac{1}{\sqrt{4 \pi \omega}} e^{-i \omega \bar{v}(v)}, \quad \phi_{2, \omega}(u) \equiv \frac{1}{\sqrt{4 \pi \omega}} e^{-i \omega \bar{u}(u)},
$$

we obtain the same normal component of the current as for the respective fermionic function. We can establish the following analogy

$$
\psi_{j, \omega} \longleftrightarrow \phi_{j, \omega}
$$

and using this analogy we can construct the $\bar{g}^{i n}$ modes in the fermionic case as follows: simply replace in formulas (23) and (24) the functions $\phi_{j, \omega}$ with the functions $\psi_{j, \omega}$, to obtain

$$
\begin{aligned}
\bar{g}_{\omega, R}^{i n}(u, v)= & \frac{1}{\sqrt{2 \pi}} s(\omega)\left(\begin{array}{l}
0 \\
1
\end{array}\right) \sqrt{\bar{v}^{\prime}(v)} e^{-i \omega \bar{v}(v)} \theta(u-U(v)) \\
& +\frac{1}{\sqrt{2 \pi}}\left[\left(\begin{array}{l}
0 \\
1
\end{array}\right) \sqrt{\bar{v}^{\prime}(v)} e^{-i \omega \bar{v}(v)}+r(\omega)\left(\begin{array}{l}
1 \\
0
\end{array}\right) \sqrt{\bar{u}^{\prime}(u)} e^{-i \omega \bar{u}(u)}\right] \theta(v-V(u))
\end{aligned}
$$

and

$$
\begin{aligned}
\bar{g}_{\omega, L}^{i n}(u, v)= & \frac{1}{\sqrt{2 \pi}}\left[\left(\begin{array}{l}
1 \\
0
\end{array}\right) \sqrt{\bar{u}^{\prime}(u)} e^{-i \omega \bar{u}(u)}+r(\omega)\left(\begin{array}{l}
0 \\
1
\end{array}\right) \sqrt{\bar{v}^{\prime}(v)} e^{-i \omega \bar{v}(v)}\right] \theta(u-U(v)) \\
& +\frac{1}{\sqrt{2 \pi}} s(\omega)\left(\begin{array}{l}
1 \\
0
\end{array}\right) \sqrt{\bar{u}^{\prime}(u)} e^{-i \omega \bar{u}(u)} \theta(u-U(v)) .
\end{aligned}
$$

As in the scalar case, the modes $\bar{g}^{i n}$ are not the 'in' modes in the coordinates $(u, v)$. However using the fact that the modes $\bar{g}^{i n}$ constitute an orthonormal basis of the space of solutions, we can obtain the following expression of the 'in' modes:

$$
\psi_{\omega, k}^{i n}=\int_{\mathbb{R}} d \omega^{\prime}\left(\bar{g}_{\omega^{\prime}, k}^{i n} ; \psi_{\omega, k}^{i n}\right) \bar{g}_{\omega^{\prime}, k}^{i n}
$$

If we choose the coefficients $r(w)=\frac{-i \alpha}{\omega+i \alpha}$ and $s(w)=\frac{\omega}{\omega+i \alpha}$, with $\alpha \geq 0$, on the right side of mirror we have

$$
\psi_{\omega, R}^{i n}(u, v)=\frac{1}{\sqrt{2 \pi}}\left(\begin{array}{c}
0 \\
1
\end{array}\right) e^{-i \omega v}+\psi_{\omega, R}^{r e f l}(u), \quad \psi_{\omega, L}^{i n}(u, v)=\psi_{\omega, L}^{\text {trans }}(u)
$$

where

$$
\begin{aligned}
& \left\{\begin{array}{ccc}
\frac{-i \alpha \sqrt{V^{\prime}(u)}}{\omega+i \alpha} e^{-i \omega V(u)} ; & u \leq 0 \\
\frac{-i \alpha \sqrt{\bar{u}^{\prime}(u)}}{\omega+i \alpha} & e^{-\alpha \bar{u}(u)} & u \leq 0
\end{array}\right. \\
& \psi_{\omega, R}^{r e f l}(u)=\frac{1}{\sqrt{2 \pi}}\left(\begin{array}{l}
1 \\
0
\end{array}\right)\left\{\quad-\frac{2 \alpha e^{-i \frac{\omega}{k}} \sqrt{\bar{u}^{\prime}(u)}}{k} \int_{0}^{\frac{k}{2} \bar{u}(u)} d s \sqrt{s+1-\frac{k}{2} \bar{u}(u)} e^{\frac{i \omega}{k}\left(s+1-\frac{k}{2} \bar{u}(u)\right)^{2}} e^{-\frac{2 \alpha s}{k}} ; \quad 0 \leq u \leq u_{0}\right. \\
& \frac{-i \alpha \sqrt{\bar{u}^{\prime}(u)}}{\omega+i \alpha} e^{-\alpha \bar{u}(u)}-\frac{i \alpha \sqrt{V^{\prime}(u)}}{\sqrt{A} \omega+i \alpha}\left[e^{-i \omega V(u)}-e^{-i \omega V\left(u_{0}\right)} e^{-\alpha\left(\bar{u}(u)-\bar{u}\left(u_{0}\right)\right)}\right] \\
& -\frac{2 \alpha e^{-i \frac{\omega}{k}} \sqrt{\bar{u}^{\prime}(u)} e^{-\alpha\left(\bar{u}(u)-\bar{u}\left(u_{0}\right)\right)}}{k} \int_{0}^{\frac{k}{2} \bar{u}\left(u_{0}\right)} d s \sqrt{s+1-\frac{k}{2} \bar{u}\left(u_{0}\right)} e^{\frac{i \omega}{k}\left(s+1-\frac{k}{2} \bar{u}\left(u_{0}\right)\right)^{2}} e^{-\frac{2 \alpha s}{k}} ; \quad u \geq u_{0}
\end{aligned}
$$


and

$$
\psi_{\omega, L}^{\text {trans }}(u)=\frac{1}{\sqrt{2 \pi}}\left(\begin{array}{l}
1 \\
0
\end{array}\right)\left\{\begin{array}{c}
\frac{\omega \sqrt{V^{\prime}(u)}}{\omega+i \alpha} e^{-i \omega V(u)} ; \\
\frac{-i \alpha \sqrt{\bar{u}^{\prime}(u)}}{\omega+i \alpha} e^{-\alpha \bar{u}(u)}+e^{-i \omega u} \\
-\frac{2 \alpha \sqrt{\bar{u}^{\prime}(u)}}{k} \int_{0}^{\frac{k}{2} \bar{u}(u)} d s\left(s+1-\frac{k}{2} \bar{u}(u)\right)^{2 i \omega / k-1 / 2} e^{-2 \alpha s / k} ; \\
\frac{-i \alpha \sqrt{\bar{u}^{\prime}(u)}}{\omega+i \alpha} e^{-\alpha \bar{u}(u)}+\frac{e^{-i \omega u_{0}}}{\omega+i \alpha \sqrt{A}}\left[\omega e^{-i \frac{\omega}{\sqrt{A}}\left(\bar{u}(u)-\bar{u}\left(u_{0}\right)\right)}+i \alpha \sqrt{A} e^{\left.-\alpha \sqrt{A}\left(\bar{u}(u)-\bar{u}\left(u_{0}\right)\right)\right]}\right. \\
-\frac{2 \alpha \sqrt{\bar{u}^{\prime}(u)}}{k} e^{-\alpha\left(\bar{u}(u)-\bar{u}\left(u_{0}\right)\right)} \int_{0}^{\frac{k}{2} \bar{u}\left(u_{0}\right)} d s\left(s+1-\frac{k}{2} \bar{u}\left(u_{0}\right)\right)^{2 i \omega / k-1 / 2} e^{-2 \alpha s / k} . \\
u \geq u_{0}
\end{array}\right.
$$

Note that in the case of perfect reflection, that is, when $\alpha \rightarrow \infty$, we have

$$
\psi_{\omega, R}^{r e f l}(u) \rightarrow-\sqrt{\frac{V^{\prime}(u)}{2 \pi}}\left(\begin{array}{l}
1 \\
0
\end{array}\right) e^{-i \omega V(u)}, \quad \psi_{\omega, L}^{\text {trans }}(u) \rightarrow 0,
$$

and when the mirror is transparent, i.e., when $\alpha \rightarrow 0$, it turns out that

$$
\psi_{\omega, R}^{r e f l}(u) \rightarrow 0, \quad \psi_{\omega, L}^{\text {trans }}(u) \rightarrow \frac{1}{\sqrt{2 \pi}}\left(\begin{array}{l}
1 \\
0
\end{array}\right) e^{-i \omega u} .
$$

To calculate the production of particles on the rhs of the mirror we must obtain $\beta_{\omega, \omega^{\prime}}^{R, R} \equiv\left(\psi_{\omega, R}^{\text {out* }} ; \psi_{\omega^{\prime}, R}^{\text {in }}\right)^{*}$ and $\beta_{\omega, \omega^{\prime}}^{R, L} \equiv\left(\psi_{\omega, R}^{\text {out* }} ; \psi_{\omega^{\prime}, L}^{\text {in }}\right)^{*}$. We start by calculating $\beta_{\omega, \omega^{\prime}}^{R, R}$. Choosing the right null infinity region $\mathcal{J}_{R}^{+}$, we have $\beta_{\omega, \omega^{\prime}}^{R, R}=\int_{R}\left(\psi_{\omega, R}^{\text {out }}\right)^{t} \psi_{\omega^{\prime}, R}^{r e f l} d u$, then, for $1 \ll \omega^{\prime} / k \ll e^{k u_{0}}$ and $1 \ll \omega^{\prime} / \omega \ll e^{k u_{0}}$, we get

$$
\begin{aligned}
\beta_{\omega, \omega^{\prime}}^{R, R} \cong & \frac{1}{2 \pi} \frac{\alpha}{\omega^{\prime}+i \alpha} \frac{1}{\omega+\omega^{\prime}} \\
& -\frac{\alpha e^{-i \omega^{\prime} / k}}{k^{2} \pi} \int_{A}^{1} d x x^{i \omega / k-3 / 4} \int_{0}^{(1-\sqrt{x})} d s \sqrt{s+\sqrt{x}} e^{\frac{i \omega^{\prime}}{k}(s+\sqrt{x})^{2}} e^{-2 \alpha s / k} .
\end{aligned}
$$

Integrating by parts with respect to the $s$ variable, we obtain

$$
\beta_{\omega, \omega^{\prime}}^{R, R} \cong-\frac{\alpha}{2 \pi i \omega k}\left(\frac{i k}{\omega^{\prime}}\right)^{i \omega / k+1} \Gamma(1+i \omega / k),
$$

and thus, in the case of a partially reflecting mirror, it turns out that

$$
\left|\beta_{\omega, \omega^{\prime}}^{R, R}\right|^{2} \cong \frac{1}{2 \pi \omega k}\left(\frac{\alpha}{\omega^{\prime}}\right)^{2}\left(e^{2 \pi \omega / k}-1\right)^{-1} .
$$

Finally, a simple but rather cumbersome calculation yields the result we were looking for

$$
\left|\beta_{\omega, \omega^{\prime}}^{R, L}\right|^{2} \sim \frac{1}{\omega \omega^{\prime}} \mathcal{O}\left[\left(\frac{\alpha}{\omega^{\prime}}\right)^{2}\right]
$$

\section{CONCLUSIONS}

In this paper we have studied in detail the creation of scalar massless particles in a two-dimensional Minkowski spacetime (the Davies-Fulling theory) and specifically for the case of a semi-transparent mirror, which reflects low frequency modes but is transparent to high enough frequencies, being the reflection and transmission coefficients analytic functions of the frequency. The considered mirror is initially at rest, then accelerates, during some finite time, along a trajectory 
that simulates a black hole collapse (as defined by Walker [5], and Carlitz and Willey [6]), and finally rests moving with constant velocity.

When the reflection and transmission coefficients are those in the model proposed by Barton, Calogeracos, and Nicolaevici [10, 11, 12], namely $r(w)=-i \alpha /(\omega+i \alpha)$ and $s(w)=\omega /(\omega+i \alpha)$, with $\alpha \geq 0$, the Bogoliubov coefficients on the back side of the mirror could be computed exactly. This has allowed us to rigorously prove that, when $\alpha$ is very large (the case of an ideal, perfectly reflecting mirror) a thermal emission of scalar massless particles obeying Bose-Einstein statistics is radiated from the mirror (a black body radiation), in accordance with previous results in the literature. Moreover, we have also seen that when $\alpha$ is finite (the case of a semi-transparent mirror, that is, a physically realistic situation) the surprising result is obtained that the thermal emission of scalar massless particles obeys Fermi-Dirac statistics. We have also shown in detail that the reverse change of statistics takes place in a bidimensional fermionic model for massless particles, namely, that the Fermi-Dirac statistics for the completely reflecting situation gives rise to the Bose-Einstein statistics for the case of a semi-transparent, physical mirror.

The results we have obtained are absolutely solid - they do not hang on a perturbative expansion or approximation of any sort. The physical reason for this surprising change of statistics may be found in the fact that the form of the spectrum is actually determined not through the statistics of the field but rather by the specific trajectory of the mirror and by its interaction with the radiation field. The same kind of phenomenon occurs in the case of an electric charge following the trajectory $v=\frac{1}{k}\left(1-e^{-k u}\right)$. When the radiation field has spin 1 , the radiation emitted by the charge obeys Bose-Einstein statistics, but when a scalar charge, and consequently an scalar radiation field, is considered, the emitted radiation will obey Fermi-Dirac statistics [14].

Another situation where this kind of features occurs, is when one measures the spectrum of an scalar field using a DeWitt detector [16, 17] which follows a uniformly accelerated world-line in Minkowski space-time. In this case when the dimension of the space-time is even the Bose-Einstein statistics is obtained. However, when the dimension is odd, precisely the reverse change of statistics occurs in the emitted radiation [15, 24, 25, 26].

Acknowledgements. This investigation is partly based on work done while on leave at the Department of Physics and Astronomy, Dartmouth College, 6127 Wilder Laboratory, Hanover, NH 03755, USA. This research was supported by MEC (Spain), projects MTM2005-07660-C02-01 and FIS2006-02842, and by AGAUR (Generalitat de Catalunya), grant 2007BE-1003 and contract 2005SGR-00790.

\section{APPENDIX}

We will here derive in detail the total number of produced particles and their energy. First, we start with the case of a perfectly reflecting mirror. Assuming that the mirror's velocity converges fast enough to some constant, when $|u| \rightarrow \infty$, we have

$$
\beta_{\omega, \omega^{\prime}}^{R, R}=\frac{1}{2 \pi} \sqrt{\frac{\omega}{\omega^{\prime}}} \int_{\mathbb{R}} d u e^{-i \omega u} e^{-i \omega^{\prime} V(u)}
$$

and, integrating by parts, we get

$$
\beta_{\omega, \omega^{\prime}}^{R, R}=-\frac{1}{2 \pi i} \sqrt{\omega \omega^{\prime}} \int_{\mathbb{R}} d u \frac{V^{\prime \prime}(u)}{\left(\omega+\omega^{\prime} V^{\prime}(u)\right)^{2}} e^{-i \omega u} e^{-i \omega^{\prime} V(u)} .
$$

For simplicity, we will assume that the mirror's acceleration is discontinuous at the point $u=a$. After another integration by parts, we obtain

$$
\begin{aligned}
\beta_{\omega, \omega^{\prime}}^{R, R} & =-\frac{1}{2 \pi} \sqrt{\omega \omega^{\prime}} \frac{1}{\left(\omega+\omega^{\prime} V^{\prime}(a)\right)^{3}} e^{-i \omega a} e^{-i \omega^{\prime} V(a)}\left(V^{\prime \prime}\left(a^{-}\right)-V^{\prime \prime}\left(a^{+}\right)\right) \\
& +\frac{1}{2 \pi} \sqrt{\omega \omega^{\prime}} \int_{\mathbb{R}} d u\left[\frac{V^{\prime \prime \prime}(u)}{\left(\omega+\omega^{\prime} V^{\prime}(u)\right)^{3}}-\frac{3 \omega^{\prime}\left(V^{\prime \prime}(u)\right)^{2}}{\left(\omega+\omega^{\prime} V^{\prime}(u)\right)^{4}}\right] e^{-i \omega u} e^{-i \omega^{\prime} V(u)} .
\end{aligned}
$$


From this expression, if we further assume that the mirror's trajectory is asymptotically inertial, that is $V^{\prime}(u)>0, \forall u \in \mathbb{R}$ (see for example [5]), it also follows that $\left|\beta_{\omega, \omega^{\prime}}^{R, R}\right|^{2}$ and $\hbar \omega\left|\beta_{\omega, \omega^{\prime}}^{R, R}\right|^{2}$ are integrable functions in the domain $[0, \infty)^{2} \backslash[0,1]^{2}$.

Now, we are interested in the production of particles in the infrared domain, that is, we want to calculate $\left|\beta_{\omega, \omega^{\prime}}^{R, R}\right|^{2}$ in $[0,1]^{2}$. We write the Bogoliubov coefficient as follows

$$
\beta_{\omega, \omega^{\prime}}^{R, R}=\frac{1}{2 \pi} \sqrt{\frac{\omega}{\omega^{\prime}}} \int_{\mathbb{R}} d u e^{-i\left(\omega+B \omega^{\prime}\right) u} e^{-i \omega^{\prime}(V(u)-B u)},
$$

with $B>0$. After integration by parts, we obtain

$$
\beta_{\omega, \omega^{\prime}}^{R, R}=-\frac{1}{2 \pi} \frac{\sqrt{\omega \omega^{\prime}}}{\omega+B \omega^{\prime}} \int_{\mathbb{R}} d u\left(V^{\prime}(u)-B\right) e^{-i \omega u} e^{-i \omega^{\prime} V(u)},
$$

and thus, if the function $\left|V^{\prime}(u)-B\right|$ is integrable in $\mathbb{R}$ for some $B>0$, it can be deduced that $\left|\beta_{\omega, \omega^{\prime}}^{R, R}\right|^{2}$ and $\hbar \omega\left|\beta_{\omega, \omega^{\prime}}^{R, R}\right|^{2}$ are integrable functions in the domain $[0,1]^{2}$.

An example of this kind of trajectories is

$$
V(u)=\left\{\begin{array}{cc}
B u & u \leq 0 \\
V(u) & 0 \leq u \leq u_{0} \\
V\left(u_{0}\right)+B\left(u-u_{0}\right) & u \geq u_{0}
\end{array}\right.
$$

However, if we are only interested in the convergence of the function $\hbar \omega\left|\beta_{\omega, \omega^{\prime}}^{R, R}\right|^{2}$ in the domain $[0,1]^{2}$, we only need trajectories that satisfy

$$
\int_{-\infty}^{0} d u\left|V^{\prime}(u)-B_{1}\right|<\infty \text { and } \int_{0}^{\infty} d u\left|V^{\prime}(u)-B_{2}\right|<\infty
$$

for some non-negative constants $B_{1}$ and $B_{2}$ (here it is important to remark that one of these constants can be zero, that is, it is not worthwhile that the trajectory be asymptotically inertial). To prove that statement, we write

$$
\beta_{\omega, \omega^{\prime}}^{R, R}=\frac{1}{2 \pi} \sqrt{\frac{\omega}{\omega^{\prime}}}\left[\int_{-\infty}^{0} d u e^{-i\left(\omega+B_{1} \omega^{\prime}\right) u} e^{-i \omega^{\prime}\left(V(u)-B_{1} u\right)}+\int_{0}^{\infty} d u e^{-i\left(\omega+B_{2} \omega^{\prime}\right) u} e^{-i \omega^{\prime}\left(V(u)-B_{2} u\right)}\right],
$$

and assume, for simplicity, that $V(0)=0$. After integration by parts, we get the expression

$$
\begin{aligned}
\beta_{\omega, \omega^{\prime}}^{R, R}= & -\frac{1}{2 \pi} \sqrt{\omega \omega^{\prime}} \frac{B_{1}-B_{2}}{\left(\omega+B_{1} \omega^{\prime}\right)\left(\omega+B_{2} \omega^{\prime}\right)}-\frac{1}{2 \pi} \frac{\sqrt{\omega \omega^{\prime}}}{\omega+B_{1} \omega^{\prime}} \int_{-\infty}^{0} d u\left(V^{\prime}(u)-B_{1}\right) e^{-i \omega u} e^{-i \omega^{\prime} V(u)} \\
& -\frac{1}{2 \pi} \frac{\sqrt{\omega \omega^{\prime}}}{\omega+B_{2} \omega^{\prime}} \int_{0}^{\infty} d u\left(V^{\prime}(u)-B_{2}\right) e^{-i \omega u} e^{-i \omega^{\prime} V(u)},
\end{aligned}
$$

which already proves the assertion.

In conclusion, we have here demonstrated that, for asymptotically inertial trajectories with continuous velocity, the radiated energy is indeed finite. However it is also possible that an infinite production of particles with very low frequency could take place (an infrared divergence). To remove this divergence we must just assume that the initial and the final mirror velocities are the same.

For completeness, we should comment on the very interesting process of particle creation, for the case of a partially transmitting mirrors. In this situation, at high enough frequency the mirror behaves as transparent, and then there is no particle production, with independence of the mirror's trajectory. On the other hand, at very low frequencies the mirror behaves as a perfect reflector, and then we have the same kind of infrared problems as for the perfectly reflecting case. As a consequence, if we are only interested in the case when the radiated energy is finite, we must restrict ourselves to consider trajectories with a continuous velocity $V^{\prime}(u), \forall u \in \mathbb{R}$, which fulfill the condition (79) as, for instance, the non-asymptotically inertial trajectory:

$$
v=V(u) \equiv\left\{\begin{array}{cl}
u & \text { if } u \leq 0 \\
\frac{1}{k}\left(1-e^{-k u}\right) & \text { if } u \geq 0
\end{array}\right.
$$


This case can be discussed along the same lines above.

[1] S.A. Fulling and P.C.W. Davies, Proc. Roy. Soc. Lond. A348, 393 (1976).

[2] P.C.W. Davies and S.A. Fulling, Proc. Roy. Soc. Lond. A356, 237 (1977).

[3] J. Haro and E. Elizalde, Phys. Rev. Lett. 97, 130401 (2006).

[4] J. Haro and E. Elizalde, Phys. Rev. D76, 065001 (2007).

[5] W.R. Walker, Phys. Rev. D31, 767 (1985).

[6] R.D. Carlitz and R.S. Willey, Phys. Rev. D36, 2327 (1987).

[7] A. Calogeracos, J. Phys. A: Math. Gen. 35, 3435 (2002).

[8] N. Nicolaevici, J. Phys. A: Math. Gen. 36, 7667 (2003).

[9] J. Haro, J. Phys. A: Math. Gen. 38, L307 (2005).

[10] G. Barton and A. Calogeracos, Ann. Phys. (NY) 238, 227 (1995).

[11] N. Nicolaevici, Class. Quantum Grav. 18, 619 (2001); 18, 2895 (2001).

[12] A. Calogeracos, J. Phys. A: Math. Gen. 35, 3415 (2002).

[13] J. Haro and E. Elizalde, J. Phys. A41, 032002 (2008).

[14] A.I. Nikishov and V.I. Ritus, J.E.T.P. 81, 615 (1995).

[15] S. Takagi, Prog. Theo. Phys. Supp. 88, 1 (1986).

[16] N.D. Birrell and C.P.W. Davies, Quantum Fields in Curved Space (Cambridge University Press, Cambridge, 1982).

[17] B.S. DeWitt, Phys. Reports 19, 295 (1975).

[18] M. Abramowitz and I.A. Stegun, Handbook of Mathematical Functions (Dover, New York, 1972).

[19] N. Obadia and R. Parentani, Phys. Rev. D64, 044019 (2001).

[20] M.-T. Jaekel and S. Reynaud, J. Phys. I1, 1395 (1991).

[21] A. Chodos, L.R. Jaffe, K. Johnson, C.B. Thorn and V.F. Weisskopf, Phys. Rev. D9, 3471 (1974).

[22] M. Horibe, Prog. Theor. Phys. 61, 661 (1979).

[23] V.I. Ritus, J. Exp. Theor. Phys. 83, 282 (1996).

[24] H. Ooguri, Phys. Rev. D33, 3573 (1986).

[25] W.G. Unruh, Phys. Rev. D34, 1222 (1986).

[26] H. Terashima, Phys. Rev. D60, 084001 (1999). 